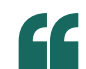

We decided to try to investigate whether these somewhat exotic looking species might really exist
NATURAL PRODUCT SYNTHESIS

\section{Tricyclic oxonium tamed}

Natural products or secondary metabolites have hugely diverse structures. Simple-looking linear precursors can, via highly reactive intermediates, fold up into a variety of complex 3D structures. Writing in the Journal of the American Chemical Society, Jonathan Burton and co-workers from the University of Oxford, in collaboration with Robert Paton at Colorado State University, describe the synthesis and characterization of tricyclic oxonium intermediates and their conversion into ten different natural products originally isolated from algae of the genus Laurencia.

The proposal of a biosynthetic pathway leading to a natural product is frequently the key to the development of a successful synthetic route, and total synthesis remains the gold standard of proof for structure assignment. Carbocationic intermediates in terpene biosyntheses are well studied; they have been invoked in the design of many natural product syntheses and have been isolated and characterized in the condensed states. Until now, however, the corresponding structures bearing a formal positive charge on oxygen (a trialkyloxonium ion) proposed for other biosyntheses have proved far more elusive.

"The structures of a number of Laurencia natural products had originally been misassigned," explains Burton. "Our work with Rob Paton on calculating the NMR spectra for the proposed structures of these natural products led us to earlier proposals of tricyclic oxonium species. We decided to try to investigate whether these somewhat exotic looking species might really exist."

In the proposed biosyntheses, the highly reactive oxonium ions result from a transannular ring closing reaction with loss of a halide ion. Burton and co-workers were able to characterize the oxonium by treatment of the precursor with a silver(I) salt. The thermodynamic driving force for the process is the formation of silver bromide, but the key to the ultimate characterization by NMR spectroscopy was the use of a large non-coordinating counterion, $\mathrm{Al}\left[\mathrm{OC}\left(\mathrm{CF}_{3}\right)_{3}\right]_{4}^{-}$, known as Krossing's anion.

Even with the use of this stabilizing anion, the oxonium ions

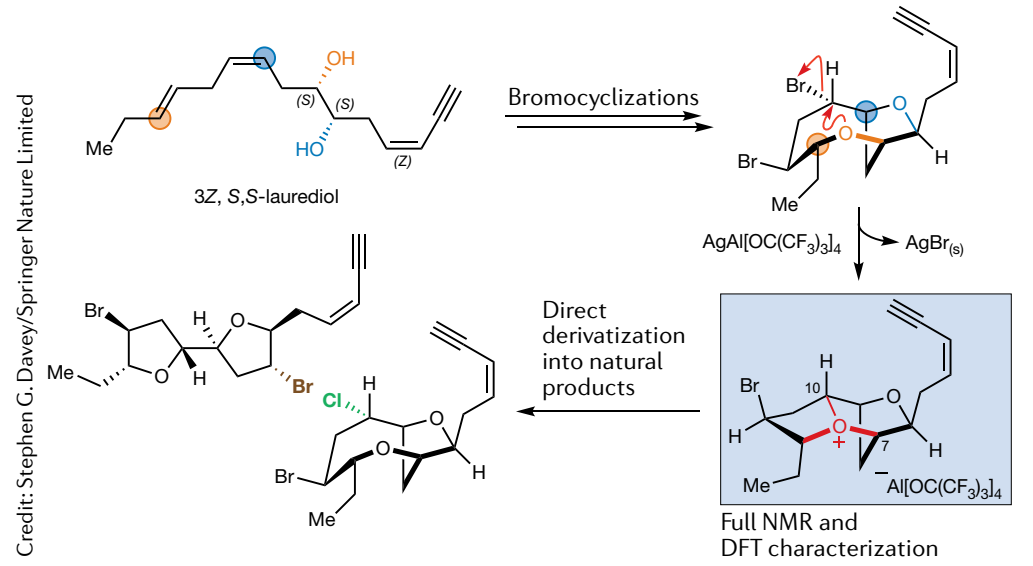

could only be characterized at low temperature. However, ${ }^{1} \mathrm{H}$ and ${ }^{13} \mathrm{C}$ NMR spectra are indicative of its formation with large downfield shifts of the signals for the three carbons adjacent to the oxygen and for the protons attached to them. In addition, a new cross-peak was observed in the ${ }^{1} \mathrm{H}-{ }^{13} \mathrm{C}$ correlation spectrum, indicating the formation of a new transannular bond. ${ }^{1} \mathrm{H}$ and ${ }^{13} \mathrm{C}$ chemical shifts calculated using density functional theory also favoured the assigned structures and formation of the oxonium ions.

Four different oxonium ions derived from the diastereomers of laurediol were prepared and characterized - the formation of one of these, from $3 Z, S, S$-laurediol is pictured. Burton and co-workers then investigated the reactions of these intermediates with a variety of nucleophiles, leading directly to ten members of the Laurencia natural product family. "Using a closely related model compound, we were also able to characterize the decomposition pathway of these oxonium ions, which we believe occurs by a 1,2-hydride shift to form an oxocarbenium ion," says Burton. Such a decomposition pathway further highlights the rich chemistry of these exotic oxonium ions.

"We'd like to continue our work to try and show that these tricyclic oxonium ions are not only chemically reasonable species but to show definitively that they are involved in the biosynthesis of these natural products," says Burton. "We'd also like to develop the oxonium ion chemistry and open up this relatively esoteric area of research to explore its full potential for organic chemistry."

Stephen G. Davey

ORIGINAL ARTICLE Chan, H. S. S. et al. Synthesis, characterization, and reactivity of complex tricyclic oxonium ions, proposed intermediates in natural product biosynthesis. J. Am. Chem. Soc. 141, 15951-15962 (2019) 